\title{
Some inequalities involving the extended gamma function and the Kummer confluent hypergeometric $k$-function
}

Kottakkaran Sooppy Nisar ${ }^{1}$, Feng Qi ${ }^{2,3,4^{*}}$ (D), Gauhar Rahmann ${ }^{5}$, Shahid Mubeen ${ }^{6}$ and Muhammad Arshad ${ }^{5}$

"Correspondence:

qifeng618@hotmail.com; qifeng618@gmail.com

${ }^{2}$ Institute of Mathematics, Henan

Polytechnic University, Jiaozuo,

China

${ }^{3}$ Department of Mathematics,

College of Science, Tianjin

Polytechnic University, Tianjin, China

Full list of author information is

available at the end of the article

\begin{abstract}
In the paper, the authors present some inequalities involving the extended gamma function and the Kummer confluent hypergeometric $k$-function via some classical inequalities such as Chebychev's inequality for synchronous (or asynchronous, respectively) mappings, give a new proof of the log-convexity of the extended gamma function by using the Hölder inequality, and introduce a Turán type mean inequality for the Kummer confluent $k$-hypergeometric function.
\end{abstract}

MSC: Primary 33B15; secondary 33C15; 33B20; 33C05; 41 A60

Keywords: Extended gamma function; Inequality; Logarithmic convexity; Confluent hypergeometric $k$-function

\section{Introduction}

The gamma function $\Gamma$ can be defined $[24,28,31,32]$ by

$$
\Gamma(z)=\int_{0}^{\infty} t^{z-1} e^{-t} \mathrm{~d} t, \quad \Re(z)>0 .
$$

Alternatively, it can also be defined [20] by

$$
\Gamma(z)=\lim _{n \rightarrow \infty} \frac{n ! n^{z-1}}{(z)_{n}},
$$

where $(z)_{n}$ for $z \neq 0$ is the Pochhammer symbol defined [27] as

$$
(z)_{n}= \begin{cases}z(z+1)(z+2) \cdots(z+n-1), & n \geq 1 \\ 1, & n=0 .\end{cases}
$$

The relation between $(z)_{n}$ and $\Gamma(z)$ is

$$
(z)_{n}=\frac{\Gamma(z+n)}{\Gamma(z)} .
$$

The beta function $B(x, y)$ can be defined $[18,21,22]$ by

$$
B(x, y)=\int_{0}^{1} t^{x-1}(1-t)^{y-1} \mathrm{~d} t, \quad \Re(x), \Re(y)>0
$$

(c) The Author(s) 2018. This article is distributed under the terms of the Creative Commons Attribution 4.0 International License (http://creativecommons.org/licenses/by/4.0/), which permits unrestricted use, distribution, and reproduction in any medium, provided you give appropriate credit to the original author(s) and the source, provide a link to the Creative Commons license, and indicate if changes were made. 
and can be expressed by

$$
B(x, y)=\frac{\Gamma(x) \Gamma(y)}{\Gamma(x+y)}, \quad \Re(x), \Re(y)>0 .
$$

In 1995, Chaudhry and Zubair [4] introduced the extended gamma function,

$$
\Gamma_{b}(z)=\int_{0}^{\infty} t^{z-1} e^{-t-b t^{-1}} \mathrm{~d} t, \quad \Re(z)>0, b \geq 0 .
$$

If $b=0$, then $\Gamma_{b}$ becomes the classical gamma function $\Gamma$.

In 1997, Chaudhry et al. [3] introduced the extended beta function,

$$
B_{b}(x, y)=\int_{0}^{1} t^{x-1}(1-t)^{y-1} e^{-b / t(1-t)} \mathrm{d} t, \quad \Re(b), \Re(x), \Re(y)>0 .
$$

It is clear that $B_{0}(x, y)=B(x, y)$.

In 2009, Barnard et al. [1] established three inequalities

$$
\begin{aligned}
& {[\phi(a, a+b, x)]^{2}>\phi(a+v, a+b, x) \phi(a-v, a+b, x),} \\
& {[\phi(a, c, x)]^{2}>\phi(a+v, c, x) \phi(a-v, c, x),}
\end{aligned}
$$

and

$$
\begin{aligned}
A(\phi(a+v, a+b, x), \phi(a-v, a+b, x)) & >\phi(a, a+b, x) \\
& >G(\phi(a+v, a+b, x), \phi(a-v, a+b, x)),
\end{aligned}
$$

where $A(\alpha, \beta)=\frac{\alpha+\beta}{2}$ and $G(\alpha, \beta)=\sqrt{\alpha \beta}$ are the arithmetic and geometric means and

$$
\phi(a, b, x)=\sum_{n=0}^{\infty} \frac{(a)_{n}}{(b)_{n}} \frac{x^{n}}{n !}
$$

is the Kummer confluent hypergeometric function [25, 28].

The Kummer confluent hypergeometric $k$-function is defined by

$$
\phi_{k}(a, b, x)=\sum_{n=0}^{\infty} \frac{(a)_{n, k}}{(b)_{n, k}} \frac{x^{n}}{n !}
$$

where

$$
(a)_{n, k}=a(a+k)(a+2 k) \cdots[a+(n-1) k]
$$

for $n \geq 1$ and $k>0$ with $(a)_{0, k}=1$ is the Pochhammer $k$-symbol, which can also be rewritten as

$$
(a)_{n, k}=\frac{\Gamma_{k}(a+n k)}{\Gamma_{k}(a)}
$$


and the gamma $k$-function $\Gamma_{k}(a)$ is defined [6] by

$$
\Gamma_{k}(a)=\int_{0}^{\infty} t^{a-1} e^{-t^{k} / k} \mathrm{~d} t
$$

In 2012, Mubeen [15] introduced the $k$-analogue of Kummer's transformation as

$$
\phi_{k}(a, b, x)=e^{x} \phi_{k}(a, b-a,-x) .
$$

In Sect. 2, we prepare two lemmas. In Sect. 3, we discuss applications of some integral inequalities such as Chebychev's integral inequality. In Sect. 4, we prove the logarithmic convexity of the extended gamma function. In the last section, we introduce a mean inequality of Turán type for the Kummer confluent hypergeometric $k$-function.

\section{Lemmas}

In order to obtain our main results, we need the following lemmas.

Lemma 2.1 (Chebychev's integral inequality $[7,8,12,23])$ Let $f, g, h: I \subseteq \mathbb{R} \rightarrow \mathbb{R}$ be mappings such that $h(x) \geq 0, h(x) f(x) g(x), h(x) f(x)$, and $h(x) g(x)$ are integrable on I. If $f(x)$ and $g(x)$ are synchronous (or asynchronous, respectively) on $I$, that is,

$$
[f(x)-f(y)][g(x)-g(y)] \gtreqless 0
$$

for all $x, y \in I$, then

$$
\int_{I} h(x) \mathrm{d} x \int_{I} h(x) f(x) g(x) \mathrm{d} x \gtreqless \int_{I} h(x) f(x) \mathrm{d} x \int_{I} h(x) g(x) \mathrm{d} x .
$$

Lemma 2.2 (Hölder's inequality $[29,30]$ ) Let $p$ and $q$ be positive real numbers such that $\frac{1}{p}+\frac{1}{q}=1$ and $f, g:[c, d] \rightarrow \mathbb{R}$ be integrable functions. Then

$$
\left|\int_{c}^{d} f(x) g(x) \mathrm{d} x\right| \leq\left[\int_{c}^{d}|f(x)|^{p} \mathrm{~d} x\right]^{1 / p}\left[\int_{c}^{d}|g(z)|^{q} \mathrm{~d} x\right]^{1 / q} .
$$

\section{Inequalities involving the extended gamma function via Chebychev's integral inequality}

In this section, we prove some inequalities involving the extended gamma function via Chebychev's integral inequality in Lemma 2.1.

Theorem 3.1 Let $m, p$ and $r$ be positive real numbers such that $p>r>-m$. If $r(p-m-r) \gtreqless$ 0 , then

$$
\Gamma_{b}(m) \Gamma_{b}(p) \gtreqless \Gamma_{b}(p-r) \Gamma_{b}(m+r) .
$$

Proof Let us define the mappings $f, g, h:[0, \infty) \rightarrow[0, \infty)$ given by

$$
f(t)=t^{p-r-m}, \quad g(t)=t^{r}, \quad \text { and } \quad h(t)=t^{m-1} e^{-t-b t^{-1}} .
$$


If $r(p-m-r) \gtreqless 0$, then we can claim that the mappings $f$ and $g$ are synchronous (asynchronous) on $(0, \infty)$. Thus, by applying Chebychev's inequality on $I=(0, \infty)$ to the functions $f, g$ and $h$ defined above, we can write

$$
\begin{aligned}
& \int_{0}^{\infty} t^{m-1} e^{-t-b t^{-1}} \mathrm{~d} t \int_{0}^{\infty} t^{p-r-m} t^{r} t^{m-1} e^{-t-b t^{-1}} \mathrm{~d} t \\
& \quad \gtreqless \int_{0}^{\infty} t^{p-r-m} t^{m-1} e^{-t-b t^{-1}} \mathrm{~d} t \int_{0}^{\infty} t^{r} t^{m-1} e^{-t-b t^{-1}} \mathrm{~d} t .
\end{aligned}
$$

This implies that

$$
\begin{aligned}
& \int_{0}^{\infty} t^{m-1} e^{-t-b t^{-1}} \mathrm{~d} t \int_{0}^{\infty} t^{p-1} e^{-t-b t^{-1}} \mathrm{~d} t \\
& \quad \gtreqless \int_{0}^{\infty} t^{p-r-1} e^{-t-b t^{-1}} \mathrm{~d} t \int_{0}^{\infty} t^{m+r-1} e^{-t-b t^{-1}} \mathrm{~d} t .
\end{aligned}
$$

By (1.1), we acquire the required inequality (3.1).

Corollary 3.1 If $p>0$ and $q \in \mathbb{R}$ with $|q|<p$, then

$$
\Gamma_{b}(p) \leq\left[\Gamma_{b}(p-q) \Gamma_{b}(p+q)\right]^{1 / 2} .
$$

Proof By setting $m=p$ and $r=q$ in Theorem 3.1, we obtain $r(p-m-r)=-q^{2} \leq 0$ and then the inequality (3.1) provides the desired Corollary 3.1 .

Theorem 3.2 If $m, n>0$ are similarly (oppositely) unitary, then

$$
\Gamma_{b}(m+n+b) \gtreqless \frac{\Gamma_{b}(m+b+1) \Gamma_{b}(n+b+1)}{\Gamma_{b}(b+2)} .
$$

Proof Consider the mappings $f, g, h:[0, \infty) \rightarrow[0, \infty)$ defined by

$$
f(t)=t^{m-1}, \quad g(t)=t^{n-1}, \quad \text { and } \quad h(t)=t^{b+1} e^{-t-b t^{-1}} .
$$

Now if the condition $(m-1)(n-1) \gtreqless 0$ holds, then Chebychev's integral inequality applied to the functions $f, g$, and $h$ means

$$
\begin{aligned}
& \int_{0}^{\infty} t^{b+1} e^{-t-b t^{-1}} \mathrm{~d} t \int_{0}^{\infty} t^{m-1} t^{n-1} t^{b+1} e^{-t-b t^{-1}} \mathrm{~d} t \\
& \quad \gtreqless \int_{0}^{\infty} t^{m-1} t^{b+1} e^{-t-b t^{-1}} \mathrm{~d} t \int_{0}^{\infty} t^{n-1} t^{b+1} e^{-t-b t^{-1}} \mathrm{~d} t .
\end{aligned}
$$

This implies that

$$
\begin{gathered}
\int_{0}^{\infty} t^{b+1} e^{-t-b t^{-1}} \mathrm{~d} t \int_{0}^{\infty} t^{m+n+b-1} e^{-t-b t^{-1}} \mathrm{~d} t \\
\gtreqless \int_{0}^{\infty} t^{m+b} e^{-t-b t^{-1}} \mathrm{~d} t \int_{0}^{\infty} t^{n+b} e^{-t-b t^{-1}} \mathrm{~d} t .
\end{gathered}
$$


By the definition of the extended gamma function, we have

$$
\Gamma_{b}(b+2) \Gamma_{b}(m+n+b) \gtreqless \Gamma_{b}(m+b+1) \Gamma_{b}(n+b+1),
$$

or

$$
\Gamma_{b}(m+n+b) \gtreqless \frac{\Gamma_{b}(m+b+1) \Gamma_{b}(n+b+1)}{\Gamma_{b}(b+2)} .
$$

The required proof is complete.

Corollary 3.2 If $b=0$, then

$$
\Gamma(m+n) \gtreqless m n \Gamma(m) \Gamma(n) .
$$

Theorem 3.3 If $m$ and $n$ are positive real numbers such that $m$ and $n$ are similarly (oppositely) unitary, then

$$
\Gamma_{b}(b+1) \Gamma_{b}(m+n+b+1) \gtreqless \Gamma_{b}\left((m+b+1) \Gamma_{b}(n+b+1), \quad b \geq 0 .\right.
$$

Proof Consider the mappings $f, g, h:[0, \infty) \rightarrow[0, \infty)$ defined by

$$
f(t)=t^{m}, \quad g(t)=t^{n}, \quad \text { and } \quad h(t)=t^{b} e^{-t-b t^{-1}} .
$$

If the conditions of Theorem 3.1 hold, then the mappings $f$ and $g$ are synchronous (asynchronous) on $[0, \infty)$. Thus, by applying Chebychev's integral inequality in Lemma 2.1 to the functions $f, g$ and $h$ defined above, we have

$$
\begin{aligned}
& \int_{0}^{\infty} t^{b} e^{-t-b t^{-1}} \mathrm{~d} t \int_{0}^{\infty} t^{m} t^{n} t^{b} e^{-t-b t^{-1}} \mathrm{~d} t \\
& \gtreqless \int_{0}^{\infty} t^{m} t^{b} e^{-t-b t^{-1}} \mathrm{~d} t \int_{0}^{\infty} t^{n} t^{b} e^{-t-b t^{-1}} \mathrm{~d} t .
\end{aligned}
$$

This implies that

$$
\begin{aligned}
& \int_{0}^{\infty} t^{b} e^{-t-b t^{-1}} \mathrm{~d} t \int_{0}^{\infty} t^{m+n+b} e^{-t-b t^{-1}} \mathrm{~d} t \\
& \gtreqless \int_{0}^{\infty} t^{m+b} e^{-t-b t^{-1}} \mathrm{~d} t \int_{0}^{\infty} t^{n+b} e^{-t-b t^{-1}} \mathrm{~d} t .
\end{aligned}
$$

Thus by the definition of extended gamma function, we have

$$
\Gamma_{b}(b+1) \Gamma_{b}(m+n+b+1) \gtreqless \Gamma_{b}(m+b+1) \Gamma_{b}(n+b+1) .
$$

The required proof is complete.

Corollary 3.3 If $b=0$, then

$$
\Gamma(m+n) \gtreqless \frac{m n \Gamma(m) \Gamma(n)}{m+n} .
$$




\section{Log-convexity of the extended gamma function}

It is well known that, if $f>0$ and $\ln f$ is convex, then $f$ is said to be a logarithmically convex function. Every logarithmically convex must be convex. See [16] and [19, Remark 1.9]. In this section, we verify the log-convexity of extended gamma function.

Theorem 4.1 The extended gamma function $\Gamma_{b}:(0, \infty) \rightarrow \mathbb{R}$ is logarithmically convex.

Proof Let $p$ and $q$ be positive numbers such that $\frac{1}{p}+\frac{1}{q}=1$. Since

$$
\Gamma_{b}\left(\frac{x}{p}+\frac{y}{q}\right) \leq\left[\Gamma_{b}(x)\right]^{1 / p}\left[\Gamma_{b}(y)\right]^{1 / q},
$$

see [5], letting $\lambda=\frac{1}{p}$ and $(1-\lambda)=\frac{1}{q}$ leads to

$$
\Gamma_{b}[\lambda x+(1-\lambda) y] \leq\left[\Gamma_{b}(x)\right]^{\lambda}\left[\Gamma_{b}(y)\right]^{(1-\lambda)} .
$$

As a result, the function $\Gamma_{b}$ is logarithmically convex.

\section{A mean inequality of the Turán type for the Kummer confluent hypergeometric $\boldsymbol{k}$-function}

In this section, we present a mean inequality involving the confluent hypergeometric $k$ function. For this purpose, we consider the relation

$$
\phi_{k}(a+k, b, x)-\phi_{k}(a, b, x)=\frac{k x}{b} \phi_{k}(a+k, b+k, x), \quad k>0 .
$$

Theorem 5.1 For $a, b, k>0$ and $v \in \mathbb{N}$ with $a, b \geq v-k$, the inequality

$$
\left[\phi_{k}(a, a+b, x)\right]^{2}>\phi_{k}(a+v, a+b, x) \phi_{k}(a-v, a+b, x)
$$

is valid for all nonzero $x \in \mathbb{R}$.

First proof Assume that $x>0$. For $c \neq 0,-1,-2, \ldots$, define

$$
f_{v, k}(x)=\left[\phi_{k}(a, c, x)\right]^{2}-\phi_{k}(a+v, c, x) \phi_{k}(a-v, a+b, x)
$$

and

$$
f_{v+k, k}(x)=\phi_{k}(a, c, x)^{2}-\phi_{k}(a+v+k, c, x) \phi_{k}(a-v-k, a+b, x) .
$$

From (5.1), it follows that

$$
\begin{aligned}
f_{v+k, k}(x)-f_{v, k}(x)= & \phi_{k}(a+v, c, x) \phi_{k}(a-v, c, x) \\
& -\phi_{k}(a+v+k, c, x) \phi_{k}(a-v-k, c, x) \\
= & \phi_{k}(a-v, c, x)\left[\phi_{k}(a+v, c, x)-\phi_{k}(a+v+k, c, x)\right] \\
& +\phi_{k}(a+v+k, c, x)\left[\phi_{k}(a-v, c, x)-\phi_{k}(a-v-k, c, x)\right]
\end{aligned}
$$




$$
\begin{aligned}
= & \phi_{k}(a-v, c, x)\left(\frac{-k x}{c}\right) \phi_{k}(a+v+k, c+k, x) \\
& +\phi_{k}(a+v+k, c, x)\left(\frac{k x}{c}\right) \phi_{k}(a-v, c+k, x) \\
= & \frac{k x}{c} g_{v, k}(x),
\end{aligned}
$$

where

$$
\begin{aligned}
g_{v, k}(x)= & \phi_{k}(a+v+k, c, x) \phi_{k}(a-v, c+k, x) \\
& -\phi_{k}(a-v, c, x) \phi_{k}(a+v+k, c+k, x) .
\end{aligned}
$$

Accordingly, by the Cauchy product, we have

$$
\begin{aligned}
g_{v, k}(x)= & \sum_{s=0}^{\infty} \sum_{r=0}^{s} \frac{(a+v+k)_{s, k}(a-v)_{s-r, k}}{r !(s-r) !} \\
& \times\left[\frac{1}{(c)_{s, k}(c+k)_{s-r, k}}-\frac{1}{(c)_{s-r, k}(c+k)_{s, k}}\right] x^{s} \\
= & \sum_{s=0}^{\infty} \sum_{r=0}^{s} \frac{(a+v+k)_{s, k}(a-v)_{s-r, k}}{r !(s-r) !}\left[\frac{(c+m k)-(c+n k-m k)}{c(c+k)_{s, k}(c+k)_{s-r, k}}\right] x^{s} \\
= & \frac{k}{c} \sum_{s=0}^{\infty} \sum_{r=0}^{s} T_{s, r, k}(2 r-s) x^{s},
\end{aligned}
$$

where

$$
T_{s, r, k}=\frac{(a+v+k)_{s, k}(a-v)_{s-r, k}}{r !(s-r) !(c+k)_{s, k}(c+k)_{s-r, k}} .
$$

If $s$ is even, then

$$
\begin{aligned}
\sum_{r=0}^{s} T_{s, r, k}(2 r-s) & =\sum_{r=0}^{s / 2-1} T_{s, r, k}(2 r-s)+\sum_{r=s / 2+1}^{s} T_{s, r, k}(2 r-s) \\
& =\sum_{r=0}^{s / 2-1} T_{s, r, k}(2 r-s)+\sum_{r=0}^{s / 2-1} T_{s, s-r, k}(2(s-r)-s) \\
& =\sum_{r=0}^{\lceil(s-1) / 2\rceil}\left(T_{s, s-r, k}-T_{s, r, k}\right)(s-2 r),
\end{aligned}
$$

where $\lceil x\rceil$ denotes the ceiling function whose value is the greatest integer not more than $x$. Similarly, if $s$ is odd,

$$
\sum_{r=0}^{s} T_{s, r, k}(2 r-s)=\sum_{r=0}^{\lceil(s-1) / 2\rceil}\left(T_{s, s-r, k}-T_{s, r, k}\right)(s-2 r)
$$


Accordingly,

$$
\begin{aligned}
f_{v+k, k}(x)-f_{v, k}(x) & =\frac{k x}{c} g_{v, k}(x) \\
& =\frac{k^{2} x}{c^{2}} \sum_{s=1}^{\infty} \sum_{r=0}^{\lceil(s-1) / 2\rceil}\left(T_{s, s-r, k}-T_{s, r, k}\right)(s-2 r) x^{s} .
\end{aligned}
$$

Carefully simplifying gives

$$
\begin{aligned}
T_{s, s-r, k}-T_{s, r, k} & =\frac{(a+v+k)_{s, k}(a-v)_{s, k}-(a+v+1)_{s, k}(a-v)_{s, k}}{r !(s-r) !(c+k)_{s-r, k}(c+k)_{s, k}} \\
& =\frac{(a+v+k)_{s, k}(a-v)_{s, k}}{r !(s-r) !(c+k)_{s-r, k}(c+k)_{s, k}}\left[\frac{(a+v+k)_{s-r, k}}{(a+v+k)_{s, k}}-\frac{(a-v)_{s-r, k}}{(a-v)_{s, k}}\right] \\
& =\frac{(a+v+k)_{s, k}(a-v)_{s, k}}{r !(s-r) !(c+k)_{s-r, k}(c+k)_{s, k}}\left[h_{k}(a+v+k)-h_{k}(a-v)\right],
\end{aligned}
$$

where $h_{k}(x)=\frac{(x)_{s-r, k}}{(x)_{s, k}}$. For $x>0$ and $s-r>r$, that is, $\left[\frac{s-1}{2}\right] \geq r$, the logarithmic derivatives of $h_{k}$ is

$$
\frac{h_{k}^{\prime}(x)}{h_{k}(x)}=\psi_{k}(x+(s-r) k)-\psi_{k}(x+n k)>0
$$

where $\psi_{k}=\frac{\Gamma_{k}^{\prime}}{\Gamma_{k}}$ is the digamma $k$-function (see $[6,11,16]$ ). Hence, the function $h_{k}$ is increasing under the condition stated. This fact together with the aid of (5.3) and (5.4) yields

$$
\begin{aligned}
f_{v+k, k}(x)-f_{v, k}(x) & =\frac{k x}{c} g_{v, k}(x) \\
& =\frac{k^{2} x}{c^{2}} \sum_{s=1}^{\infty} \sum_{r=0}^{\lceil(s-1) / 2\rceil}\left(T_{s, s-r, k}-T_{s, r, k}\right)(s-2 r) x^{s}>0,
\end{aligned}
$$

where $a \geq v \geq 0, x>0, c+k>0$, and $c \neq 0$. Consequently, from (5.5), it follows that

$$
f_{v+k, k}(x)=\left[f_{v+k, k}(x)-f_{v, k}(x)\right]+\left[f_{v, k}(x)-f_{v-k, k}(x)\right]+\cdots+\left[f_{1, k}(x)-f_{0, k}(x)\right]
$$

is positive for $a \geq v \geq v-k \geq v-2 k \geq \cdots \geq 0$ and $f_{0, k}(x)=0$. Now replacing $v$ by $v-k$ shows that

$$
f_{v, k}(x)>0, \quad x>0, v \in \mathbb{N}, a \geq v-k .
$$

Therefore, the function $f_{v, k}$ is absolutely monotonic on $(0, \infty)$, that is, $f_{v, k}^{(\ell)}(x)>0$ for $\ell=$ $0,1,2, \ldots$. This proves Theorem 5.1 for the case $x>0$.

Now suppose that $x<0, a, b>0$, and $v \in \mathbb{N}$ with $a, b \geq v-k$. Since $\phi_{k}(a, c, x)$ is symmetric in $a$ and $b$, by interchanging $a$ and $b$ in Theorem 5.1, we obtain

$$
\phi_{k}(b, a+b,-x)^{2}-\phi_{k}(b+v, a+b,-x) \phi_{k}(b-v, a+b,-x)>0 .
$$


By using Kummer's transformation (1.2), we have

$$
e^{-2 x} \phi_{k}(a, a+b, x)^{2}-e^{-2 x} \phi_{k}(a-v, a+b, x) \phi_{k}(a+v, a+b, x)>0 .
$$

Thus, Theorem 5.1 also holds for $x<0$.

Second proof Since

$$
\begin{aligned}
(a)_{n, k} & =a(a+k)(a+2 k) \cdots(a+(n-1) k) \\
& =k^{n} \frac{a}{k}\left(\frac{a}{k}+1\right)\left(\frac{a}{k}+2\right) \cdots\left(\frac{a}{k}+(n-1)\right) \\
& =k^{n}\left(\frac{a}{k}\right)_{n}
\end{aligned}
$$

it follows that

$$
\phi_{k}(a, b ; x)=\sum_{n=0}^{\infty} \frac{(a)_{n, k}}{(b)_{n, k}} \frac{x^{n}}{n !}=\sum_{n=0}^{\infty} \frac{k^{n}(a / k)_{n}}{k^{n}(b / k)_{n}} \frac{x^{n}}{n !}=\phi\left(\frac{a}{k}, \frac{b}{k}, x\right) .
$$

Replacing $a$ and $b$ by $\frac{a}{k}$ and $\frac{b}{k}$, respectively, gives Theorem 5.1 .

Corollary 5.1 If $a>0$ and $c+k>0$ with $c \neq 0$, then the inequality

$$
\left[\phi_{k}(a, c, x)\right]^{2} \geq \phi_{k}(a-v, c, x) \phi_{k}(a+v, c, x)
$$

holds for any $v \in \mathbb{N}$ with $a \geq v-k$.

Proof This follows directly from the proof of Theorem 5.1 and the fact that Eq. (5.6) holds under the conditions $c+k>0$ and $c \neq 0$.

Corollary 5.2 If $v \in \mathbb{N}$ and $a, b \geq v$, then

$$
\begin{aligned}
A\left(\phi_{k}(a+v, a+b, x), \phi_{k}(a-v, a+b, x)\right) & >\phi_{k}(a, a+b, x) \\
& >G\left(\phi_{k}(a+v, a+b, x), \phi_{k}(a-v, a+b, x)\right)
\end{aligned}
$$

for all nonzero $x \in \mathbb{R}$, where $A$ and $G$ are, respectively, the arithmetic and geometric means.

Proof First assume $x \geq 0$ and $a, b \geq v$ for $v \in \mathbb{N}$. Then the left hand side inequality in (5.7) is a direct consequence of the facts that

$$
A\left((a+v)_{s, k},(a-v)_{s, k}\right)=(a)_{s, k}
$$

for $s=0,1$ and

$$
A\left((a+v)_{s, k},(a-v)_{s, k}\right)>(a)_{s, k}
$$


for $s \geq 2$. Hence, by induction, we have

$$
\begin{aligned}
A\left(\phi_{k}(a+v, a+b, x), \phi_{k}(a-v, a+b, x)\right) & =\sum_{s=0}^{\infty} \frac{A\left((a+v)_{s, k},(a-v)_{s, k}\right) x^{s}}{(a+b)_{s, k} s !} \\
& >\sum_{s=0}^{\infty} \frac{(a)_{s, k} x^{s}}{(a+b)_{s, k} s !}=\phi_{k}(a, a+b, x) .
\end{aligned}
$$

For $x \geq 0$, the right hand side inequality in (5.7) follows from taking square root of (5.2). The proof of Corollary 5.2 for $x \geq 0$ is thus complete.

Now assume $x<0$ with $a, b \geq v$. Interchanging $a$ and $b$ in (5.7) one arrives at

$$
\begin{aligned}
A\left(\phi_{k}(b+v, a+b,-x), \phi_{k}(b-v, a+b,-x)\right) & >\phi_{k}(b, a+b,-x) \\
& >G\left(\phi_{k}(b+v, a+b,-x), \phi_{k}(b-v, a+b,-x)\right) .
\end{aligned}
$$

Making use of the $k$-analogue of Kummer's transformation and the homogeneity of $A$ and $G$ acquires

$$
\begin{aligned}
e^{-x} A\left(\phi_{k}(a-v, a+b, x), \phi_{k}(a+v, a+b, x)\right) & >e^{-x} \phi_{k}(a, a+b, x) \\
& >e^{-x} G\left(\phi_{k}(a-v, a+b, x), \phi_{k}(a+v, a+b, x)\right) .
\end{aligned}
$$

Consequently, Theorem (5.7) also follows for $x<0$.

Remark 5.1 In Sect. 5, we have established a Turán type and mean inequality for $k$ analogue of the Kummer confluent hypergeometric function. If we let $k \rightarrow 1$, then we can conclude to the corresponding inequalities of the confluent hypergeometric function.

Remark 5.2 In [2], some inequalities of the Turán type for confluent hypergeometric functions of the second kind were also discovered.

Remark 5.3 By the way, we note that Refs. $[9,10,13,14,26,32,33]$ belong to the same series in which inequalities and complete monotonicity for functions involving the gamma function $\Gamma(x)$ and the logarithmic function $\ln (1+x)$ were discussed.

Remark 5.4 This paper is a slightly revised version of the preprint [17].

\section{Conclusions}

In this paper, we present some inequalities involving the extended gamma function $\Gamma_{b}(z)$ via some classical inequalities such as Chebychev's inequality for synchronous (or asynchronous, respectively) mappings, give a new proof of the log-convexity of the extended gamma function $\Gamma_{b}(z)$ by using the Hölder inequality, and introduce a Turán type mean inequality for the Kummer confluent $k$-hypergeometric function $\phi(z)$. 


\section{Competing interests}

The authors declare that they have no competing interests.

\section{Authors' contributions}

All authors contributed equally to the manuscript and read and approved the final manuscript.

\section{Author details}

1 Department of Mathematics, College of Arts and Science at Wadi Aldawaser, Prince Sattam Bin Abdulaziz University, Riyadh Region, Kingdom of Saudi Arabia. ${ }^{2}$ Institute of Mathematics, Henan Polytechnic University, Jiaozuo, China. ${ }^{3}$ Department of Mathematics, College of Science, Tianjin Polytechnic University, Tianjin, China. ${ }^{4}$ Institute of Fundamental and Frontier Sciences, University of Electronic Science and Technology of China, Chengdu, China. ${ }^{5}$ Department of Mathematics, International Islamic University, Islamabad, Pakistan. ${ }^{6}$ Department of Mathematics, University of Sargodha, Sargodha, Pakistan.

\section{Publisher's Note}

Springer Nature remains neutral with regard to jurisdictional claims in published maps and institutional affiliations.

\section{Received: 5 February 2018 Accepted: 11 May 2018 Published online: 19 June 2018}

\section{References}

1. Barnard, R.W., Gordy, M.B., Richards, K.C.: A note on Turán type and mean inequalities for the Kummer function. J. Math. Anal. Appl. 349(1), 259-263 (2009). https://doi.org/10.1016/j.jmaa.2008.08.024

2. Bhukya, R., Akavaram, V., Qi, F.: Some inequalities of the Turán type for confluent hypergeometric functions of the second kind. HAL archives (2018), available online at https://hal.archives-ouvertes.fr/hal-01701854

3. Chaudhry, M.A., Qadir, A., Rafique, M., Zubair, S.M.: Extension of Euler's beta function. J. Comput. Appl. Math. 78(1), 19-32 (1997). https://doi.org/10.1016/S0377-0427(96)00102-1

4. Chaudhry, M.A., Zubair, S.M.: On the decomposition of generalized incomplete gamma functions with applications of Fourier transforms. J. Comput. Appl. Math. 59(3), 253-284 (1995). https://doi.org/10.1016/0377-0427(94)00026-W

5. Chaudhry, M.A., Zubair, S.M.: On a Class of Incomplete Gamma Functions with Applications. Chapman \& Hall/CRC, Boca Raton (2002)

6. Díaz, R., Pariguan, E.: On hypergeometric functions and Pochhammer k-symbol. Divulg. Mat. 15(2), 179-192 (2007)

7. Dragomir, S.S., Agarwal, R.P., Barnett, N.S.: Inequalities for beta and gamma functions via some classical and new integral inequalities. J. Inequal. Appl. 5(2), 103-165 (2000). https://doi.org/10.1155/S1025583400000084

8. Dragomir, S.S., Wang, S.: Applications of Ostrowski's inequality to the estimation of error bounds for some special means and for some numerical quadrature rules. Appl. Math. Lett. 11(1), 105-109 (1998). https://doi.org/10.1016/S0893-9659(97)00142-0

9. Guo, B.-N., Qi, F.: Monotonicity of functions connected with the gamma function and the volume of the unit ball. Integral Transforms Spec. Funct. 23(9), 701-708 (2012). https://doi.org/10.1080/10652469.2011.627511

10. Ivády, P.: A note on a gamma function inequality. J. Math. Inequal. 3(2), 227-236 (2009). https://doi.org/10.7153/jmi-03-23

11. Kokologiannaki, C.G.: Properties and inequalities of generalized k-gamma, beta and zeta functions. Int. J. Contemp. Math. Sci. 5(13-16), 653-660 (2010)

12. Kumar, P., Singh, S.P., Dragomir, S.S.: Some inequalities involving beta and gamma functions. Nonlinear Anal. Forum 6(1), 143-150 (2001)

13. Kupán, P.A., Márton, G., Szász, R.: A result regarding monotonicity of the Gamma function. Acta Univ. Sapientiae Math. 9(2), 291-302 (2017). https://doi.org/10.1515/ausm-2017-0022

14. Kupán, P.A., Szász, R.: Monotonicity theorems and inequalities for the gamma function. Math. Inequal. Appl. 17(1), 149-159 (2014). https://doi.org/10.7153/mia-17-11

15. Mubeen, S.: k-Analogue of Kummer's first formula. J. Inequal. Spec. Funct. 3(3), 41-44 (2012)

16. Nantomah, K., Prempeh, E.: The k-analogues of some inequalities for the digamma function. Math. Æterna 4(3-4), 269-273 (2014)

17. Nisar, K.S., Qi, F., Rahman, G., Mubeen, S., Arshad, M.: Some inequalities involving the extended gamma and beta functions. HAL archives. https://hal.archives-ouvertes.fr/hal-01701647. (2018)

18. Olver, F.W.J., Lozier, D.W., Boisvert, R.F., Clark, C.W. (eds.): NIST Handbook of Mathematical Functions. Cambridge University Press, New York (2010). Available online at http://dlmf.nist.gov/

19. Qi, F.: Bounds for the ratio of two gamma functions. J. Inequal. Appl. 2010, Article ID 493058 (2010). https://doi.org/10.1155/2010/493058

20. Qi, F.: Limit formulas for ratios between derivatives of the gamma and digamma functions at their singularities. Filomat 27(4), 601-604 (2013). https://doi.org/10.2298/FIL1304601Q

21. Qi, F.: Parametric integrals, the Catalan numbers, and the beta function. Elem. Math. 72(3), 103-110 (2017). https://doi.org/10.4171/EM/332

22. Qi, F., Akkurt, A., Yildirim, H.: Catalan numbers, k-gamma and k-beta functions, and parametric integrals. J. Comput. Anal. Appl. 25(6), 1036-1042 (2018)

23. Qi, F., Cui, L.-H., Xu, S.-L.: Some inequalities constructed by Tchebysheff's integral inequality. Math. Inequal. Appl. 2(4), 517-528 (1999). https://doi.org/10.7153/mia-02-42

24. Qi, F., Guo, B.-N.: Integral representations and complete monotonicity of remainders of the Binet and Stirling formulas for the gamma function. Rev. R. Acad. Cienc. Exactas Fís. Nat., Ser. A Mat. 111(2), 425-434 (2017). https://doi.org/10.1007/s13398-016-0302-6

25. Qi, F., Guo, B.-N.: On the sum of the Lah numbers and zeros of the Kummer confluent hypergeometric function. Acta Univ. Sapientiae Math. (2018). In press. ResearchGate Research (2015). https://doi.org/10.13140/RG.2.1.4089.9683 
26. Qi, F., Li, W.-H.: Integral representations and properties of some functions involving the logarithmic function. Filomat 30(7), 1659-1674 (2016). https://doi.org/10.2298/FIL1607659Q

27. Qi, F., Shi, X.-T., Liu, F.-F.: Several identities involving the falling and rising factorials and the Cauchy, Lah, and Stirling numbers. Acta Univ. Sapientiae Math. 8(2), 282-297 (2016). https://doi.org/10.1515/ausm-2016-0019

28. Rainville, E.D.: Special Functions. Macmillan Co., New York (1960)

29. Rudin, W.: Real and Complex Analysis, 3rd edn. McGraw-Hill, New York (1987)

30. Tian, J.-F., Ha, M.-H.: Properties of generalized sharp Hölder's inequalities. J. Math. Inequal. 11(2), $511-525$ (2017). https://doi.org/10.7153/jmi-11-42

31. Yang, Z.-H., Tian, J.-F.: Monotonicity and inequalities for the gamma function. J. Inequal. Appl. 2017, 317 (2017). https://doi.org/10.1186/s13660-017-1591-9

32. Yang, Z.-H., Tian, J.-F.: Monotonicity and sharp inequalities related to gamma function. J. Math. Inequal. 12(1), 1-22 (2018). https://doi.org/10.7153/jmi-2018-12-01

33. Zhao, J.-L., Guo, B.-N., Qi, F.: A refinement of a double inequality for the gamma function. Publ. Math. (Debr.) 80(3-4), 333-342 (2012). https://doi.org/10.5486/PMD.2012.5010

Submit your manuscript to a SpringerOpen ${ }^{\circ}$ journal and benefit from:

- Convenient online submission

- Rigorous peer review

- Open access: articles freely available online

- High visibility within the field

- Retaining the copyright to your article

Submit your next manuscript at $\boldsymbol{~ s p r i n g e r o p e n . c o m ~}$ 